\title{
COMPACTNESS OF SUPPORT OF SOLUTIONS FOR SOME CLASSES OF NONLINEAR ELLIPTIC AND PARABOLIC SYSTEMS
}

\author{
M. BOUCHEKIF
}

Abstract

In this paper, we obtain some existence Theorems of nonnegative solutions with compact support for homogeneous Dirichlet elliptic problems; we extend also these results to parabolic systems.

Supersolution and comparison principles are our main ingredients.

\section{Introduction}

This paper is concerned with the existence of nonnegative solutions with compact support in $X:=W_{0}^{1, p}(\Omega) \cap L^{\infty}(\Omega) \times W_{0}^{1, q}(\Omega) \cap L^{\infty}(\Omega)$ for the following systems:

$$
(\mathcal{S}) \begin{cases}-\Delta_{p} u+a|u|^{\alpha-1} u=f(x, u, v) & \text { in } \Omega \\ -\Delta_{q} v+b|v|^{\beta-1} v=g(x, u, v) & \text { in } \Omega \\ u=v=0 & \text { on } \partial \Omega\end{cases}
$$

and next

$$
(\mathcal{P}) \begin{cases}\frac{\partial u}{\partial t}-\Delta_{p} u+a|u|^{\alpha-1} u=f(x, u, v) & \text { in } \Omega \times \mathbb{R}^{+} \\ \frac{\partial v}{\partial t}-\Delta_{q} v+b|v|^{\beta-1} v=g(x, u, v) & \text { in } \Omega \times \mathbb{R}^{+} \\ u(x, t)=v(x, t)=0 & \text { on } \partial \Omega \times \mathbb{R}^{+} \\ u(., 0)=u_{0}(.) & \text { in } \Omega \\ v(., 0)=v_{0}(.) & \text { in } \Omega,\end{cases}
$$

where $p>1, q>1, a, b, \alpha$ and $\beta$ are positive constants; the operator $\Delta_{p} u$, defined by $\Delta_{p} u:=\operatorname{div}\left(|\nabla u|^{p-2} \nabla u\right), p>1$ is the well known " $p$ Laplacian"; $f$ and $g$ are nonnegative Caratheodory functions and $u_{0}$ and $v_{0}$ are some given functions. 
During recent years, many papers are devoted to the study of reactiondiffusion systems which arise very often in applications such as, mathematical biology, chemical reactions and combustion theory. An excellent overview of the subject is the survey of [1].

Díaz and Herrero [4] and [5], study the case of a single equation of the form:

$$
\left(\mathcal{E}_{a, f, g}\right) \begin{cases}-\Delta_{p} u+a|u|^{\alpha-1} u=f & \text { in } \Omega \\ u=g & \text { on } \partial \Omega,\end{cases}
$$

where $a$ is a positive constant, $f \in L^{\infty}(\Omega), g \in W^{1, p}(\Omega)$ and $g_{\mid \partial \Omega} \in$ $L^{\infty}(\partial \Omega)$, both with compact support. Then, a necessary and sufficient condition for the existence of a solution $u \in W^{1, p}(\Omega) \cap L^{\infty}(\Omega)$ of $\left(\mathcal{E}_{a, f, g}\right)$ with compact support is $0<\alpha<p-1$. They obtain the same results for the associated parabolic problem.

Here we generalize the above results to some elliptic and parabolic systems and we used the iterative method based on the Comparison Principle for the problem $(\mathcal{P})$, taking in account the construction of subsuper solution introduced in [11].

Our paper is organized as follows:

1. Introduction; 2. Preliminaries; 3. Elliptic systems and, 4. Parabolic systems.

\section{Preliminaries}

We shall use the following notations:

For $p \in] 1,+\infty\left[, p^{*}\right.$ is defined by $\frac{1}{p}+\frac{1}{p^{*}}=1$.

For $a>0, p>1$ and $0<\alpha<p-1$, set

$$
K(a, p, \alpha):=\left[\frac{a(p-\alpha-1)^{p}}{p^{p-1}(p \alpha+N(p-\alpha-1))}\right]^{\frac{1}{(p-\alpha-1)}}
$$

For $T>0$ and $R_{0}>0$ and $R_{i}>0$ such that $R_{0}<R_{i}$ for $(i=1,2)$.

Consider the following sets:

$$
\begin{aligned}
D_{0} & :=B\left(R_{0}\right), \\
D_{i} & :=B\left(R_{i}\right) \backslash B\left(R_{0}\right), \\
D_{i}^{\prime} & :=\bar{B}\left(R_{i}\right)^{c} \text { for } i=1,2 ; \\
\tilde{\Omega} & :=\Omega \cap B\left(\max \left(R_{1}, R_{2}\right)+1\right), \\
Q_{T} & :=\Omega \times[0, T] \text { and } \\
\Sigma_{T} & :=\partial \tilde{\Omega} \times[0, T]
\end{aligned}
$$


where $B(R):=\{x \in \bar{\Omega} /|x|<R\}$ and $A^{c}$ is the complement any set of the $A$.

\section{Definitions.}

I) A pair $(\tilde{u}, \tilde{v})-(\hat{u}, \hat{v})$ is said to be a weak sub-super solution for the Dirichlet problem $(\mathcal{S})$ if the following conditions are satisfied:

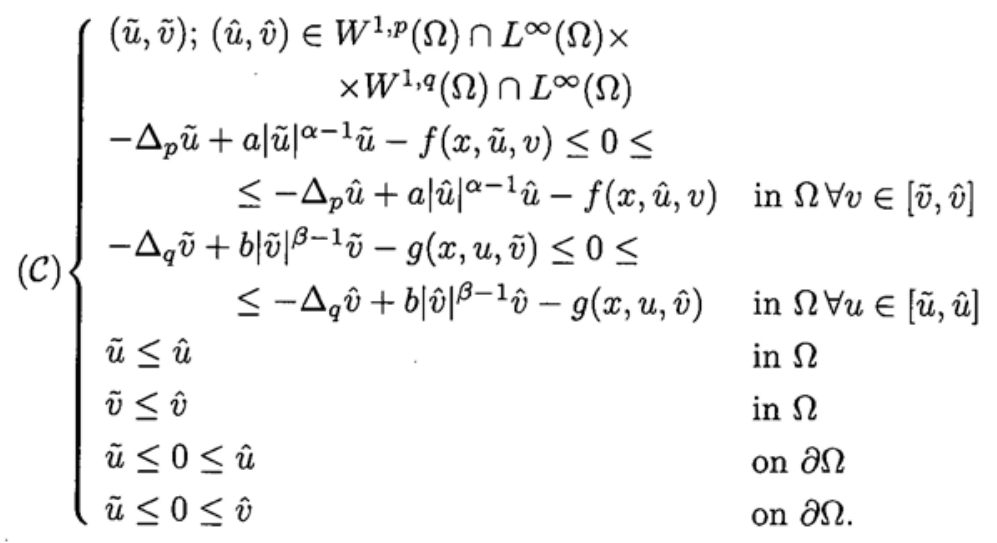

Similar definitions can be found in Díaz-Hernández [3], Hernández [7].

II) In the case of parabolic problem we consider only sub-super solutions which do not depent on $t$. Such a pair $(\tilde{u}, \tilde{v})-(\hat{u}, \hat{v})$ is sub-super solutions of $(\mathcal{P})$ if the following conditions are satisfied:
a) $(\mathcal{C})$
b) $\tilde{u}(x) \leq u_{0}(x) \leq \hat{u}(x)$ in $\Omega$,
c) $\tilde{v}(x) \leq v_{0}(x) \leq \hat{v}(x)$ in $\Omega$.

In this paper we also use the following lemmas:

Lemma 1. [2, Lemma 1.6] Assume that $p>1$ and $0<\alpha<p-1$. Then the function $u(r)=K r^{\frac{p}{p-\alpha-1}}$, where $K=K(a, p, \alpha)$ defined by (2.1), satisfies the following equation: $\frac{-1}{r^{N-1}} \frac{d}{d r}\left(r^{N-1}\left|\frac{d u}{d r}\right|^{p-2} \frac{d u}{d r}\right)+$ $a|u(r)|^{\alpha-1} u(r)=0$.

Lemma 2. [10] Suppose $\mu$ and $\nu$ are in $\mathbb{R}^{N}$. Then there exists $C>0$ such that:

$$
\begin{cases}\left(|\mu|^{p-2} \mu-|\nu|^{p-2} \nu\right)(\mu-\nu) \geq C|\mu-\nu|^{p} & \text { if } p \geq 2 \\ \left(|\mu|^{p-2} \mu-|\nu|^{p-2} \nu\right)(\mu-\nu) \geq C \frac{(|\mu-\nu|)^{2}}{(|\mu|+|\nu|)^{2-p}} & \text { if }|\mu|+|\nu| \neq 0 \\ & \text { and } 1<p \leq 2 .\end{cases}
$$


Lemma 3. [8] Let $Y$ be a Banach space.

If $u \in L^{p}(0, T ; Y)$ and $\frac{\partial u}{\partial t} \in L^{p}(0, T ; Y)(1 \leq p \leq+\infty)$. Then after an eventual modification on a set of measure zero of $(0, T), u$ is continuous from $[0, T]$ to $Y$.

Let $R_{0}>0$ be given. We seek a sub-super solution of $(\mathcal{S})$ and $(\mathcal{P})$ in the following way. Let $(\tilde{u}, \tilde{v})=(0,0)$ and $(\hat{u}, \hat{v})$ be defined by: $\hat{u}(x)=$ $G_{1}(|x|), \hat{v}(x)=G_{2}(|x|)$ for $x \in \Omega$ where:

$$
\begin{aligned}
& G_{1}(r)= \begin{cases}-A_{1} r^{p^{*}}+B_{1} & \text { if } r \leq R_{0} \\
K_{1}\left(R_{1}-r\right)^{\frac{p}{p-\alpha-1}} & \text { if } R_{0} \leq r \leq R_{1} \\
0 & \text { if } r \geq R_{1},\end{cases} \\
& G_{2}(r)= \begin{cases}-A_{2} r^{q^{*}}+B_{2} & \text { if } r \leq R_{0} \\
K_{2}\left(R_{2}-r\right)^{\frac{q}{q-\beta-1}} & \text { if } R_{0} \leq r \leq R_{2} \\
0 & \text { if } r \geq R_{2}\end{cases}
\end{aligned}
$$

with $K_{1}:=K(a, p, \alpha) ; K_{2}:=K(b, q, \beta)$ (defined by $\left.(2.1)\right), A_{i}, B_{i}$ and $R_{i}(i=1,2)$ are some positive constants.

First we need that $\hat{u} \in \mathcal{C}^{1}(\bar{\Omega})$ (resp. $\hat{v} \in \mathcal{C}^{1}(\bar{\Omega})$ ) which implies that the positive constants $A_{1}, B_{1}$ (resp. $A_{2}, B_{2}$ ) satisfy:

$$
\begin{aligned}
-A_{1} R_{0}^{p^{*}}+B_{1} & =K_{1} X_{1}^{\frac{p}{p-\alpha-1}}, \\
A_{1} p^{*} R_{0}^{p^{*}-1} & =K_{1} \frac{p}{p-\alpha-1} X_{1}^{\frac{\alpha+1}{p-\alpha-1}}, \\
-A_{2} R_{0}^{q^{*}}+B_{2} & =K_{2} X_{2}^{\frac{q}{q-\beta-1}}, \\
A_{2} q^{*} R_{0}^{q^{*}-1} & =K_{2} \frac{q}{q-\beta-1} X_{2}^{\frac{\beta+1}{q-\beta-1}},
\end{aligned}
$$

where $X_{i}=R_{i}-R_{0}$ for $i=1,2$. These constants will be completely determined in each one of the following sections.

\section{Elliptic systems}

We study the elliptic system $(\mathcal{S})$, where

$\left(\mathcal{H}_{0}\right) \Omega$ is a regular open set in $\mathbb{R}^{N}$ (not necessarly bounded); $a>0$, $b>0, p>1, q>1,0<\alpha<p-1,0<\beta<q-1$ are given numbers.

$\left(\mathcal{H}_{1}\right) f(x, u, v)$ and $g(x, u, v)$ are Caratheory functions, $f(x, ., v)$ (resp. $g(x, u,)$.$) is a nondecreasing function for fixed v$ (resp. fixed $u$ ). 
$\left(\mathcal{H}_{2}\right)$ The functions $f(x, u, v)$ and $g(x, u, v)$ satisfy:

$$
\begin{aligned}
& 0 \leq f(x, u, v) \leq c u^{\gamma} v^{\delta+1}+\varphi(x) v^{\eta}+f_{1}(x) \text { and } \\
& 0 \leq g(x, u, v) \leq d u^{\gamma+1} v^{\delta}+\psi(x) u^{\xi}+g_{1}(x) \text { for } u, v \geq 0,
\end{aligned}
$$

where

$c, d, \delta, \eta, \gamma$ and $\xi$ are nonnegative constants;

$\varphi, \psi, f_{1}$ and $g_{1}$ are nonnegative, bounded measurable functions such that:

$\operatorname{supp} f_{1} \cup \operatorname{supp} g_{1} \cup \operatorname{supp} \varphi \cup \operatorname{supp} \psi \subset B\left(R_{0}\right)$ for some $R_{0}>0$.

We seek solutions $(u, v) \in X$ satisfying $(\mathcal{S})$ in the distributional sense.

First we give some conditions on $\alpha, \beta, f$ and $g$ which insure that $(\hat{u}, \hat{v})$ defined in $(2.2)$ is a supersolution of $(\mathcal{S})$.

Proposition 1. If $\left(\mathcal{H}_{0}\right),\left(\mathcal{H}_{1}\right)$ and $\left(\mathcal{H}_{2}\right)$ are satisfied with $\gamma \geq \alpha$ and $\delta \geq \beta$, then, for sufficiently small nonnegative $c, d,\|\varphi\|_{\infty}$ and $\|\psi\|_{\infty}$, $(0,0)-(\hat{u}, \hat{v})$ is a sub-super solution for $(\mathcal{S})$ in $\Omega$.

Proof: It is obvious that $(0,0)$ is a sub solution.

By the definition of a super solution, we have to prove that:

$$
-\Delta_{p} \hat{u}+a(\hat{u})^{\alpha}-f(x, \hat{u}, v) \geq 0 \quad \forall v \in[0, \hat{v}] \text { in } \Omega .
$$

In $B\left(R_{0}\right)$, we have:

$$
-\Delta_{p} \hat{u}=N\left(A_{1} p^{*}\right)^{p-1}
$$

Then, if we take:

$$
N\left(A_{1} p^{*}\right)^{p-1} \geq c B_{1}^{\gamma} B_{2}^{\delta+1}+\|\varphi\|_{\infty} B_{2}^{\eta}+\left\|f_{1}\right\|_{\infty},
$$

$\hat{u}$ satisfies (3.1) in $B\left(R_{0}\right)$.

In $B\left(R_{1}\right) \backslash B\left(R_{0}\right), \hat{u}$ is a solution of $\left(\mathcal{E}_{\frac{a}{2}, 0,0}\right)$ by Lemma 1 [5], thus (3.1) becomes:

$$
\frac{a}{2} K_{1}^{\alpha}\left(R_{1}-r\right)^{\frac{p \alpha}{p-\alpha-1}}-c K_{1}^{\gamma} K_{2}^{\delta+1}\left(R_{1}-r\right)^{\frac{p \gamma}{p-\alpha-1}}\left(R_{2}-R_{0}\right)^{\frac{q(\delta+1)}{q-\beta-1}} \geq 0,
$$

which is satisfied if we have:

$$
\left\{\begin{array}{l}
\gamma \geq \alpha \\
c X_{1}^{\frac{p(\gamma-\alpha)}{p-\alpha-1}} X_{2}^{\frac{q(\delta+1)}{q-\beta-1}} \leq C_{1}
\end{array}\right.
$$


and analogously for the other equation of $(\mathcal{S})$, we obtain:

$$
\left\{\begin{array}{l}
\delta \geq \beta \\
d X_{1}^{\frac{p(\gamma+1)}{p-\alpha-1}} X_{2}^{\frac{q(\delta-\beta)}{q-\beta-1}} \leq C_{1}^{\prime}
\end{array}\right.
$$

where:

$C_{1}:=C_{1}(a, b ; p, q ; \gamma, \delta ; \alpha, \beta):=\frac{1}{2} a^{\frac{p-\gamma-1}{p-\alpha-1}} b^{\frac{-(\delta+1)}{q-\beta-1}} \times$

$\left[\frac{(p-\alpha-1)^{p}}{2 p^{p-1}(\alpha p+N(p-\alpha-1))}\right]^{\frac{\alpha-\gamma}{p-\alpha-1}}\left[\frac{(q-\beta-1)^{q}}{2 q^{q-1}(\beta q+N(q-\beta-1))}\right]^{-(\delta+1)} ;$

$C_{1}^{\prime}:=C_{1}(b, a ; q, p ; \delta, \gamma ; \beta, \alpha)$,

$X_{i}:=R_{i}-R_{0}$ for $i=1,2$.

So $(\hat{u}, \hat{v})$ is a supersolution of $(\mathcal{S})$ if $(2.3)$ to $(2.6),(3.2)$ to $(3.4)$ are satisfied.

From (2.3) to (2.6), (3.2) is satisfied if:

$$
\begin{array}{r}
C_{3} X_{1}^{\frac{(p-1)(\alpha+1)}{p-\alpha-1}} \geq c C_{4} X_{1}^{\frac{\gamma p}{p-\alpha-1}} X_{2}^{\frac{q(\delta+1)}{q-\beta-1}}\left(1+C_{5} X_{1}^{-1}\right)^{\gamma}\left(1+C_{5}^{\prime} X_{2}^{-1}\right)^{\delta+1}+ \\
+\|\varphi\|_{\infty} K_{2}^{\eta} X_{2}^{\frac{q \eta}{q-\beta-1}}\left(1+C_{5}^{\prime} X_{2}^{-1}\right)^{\eta}+\left\|f_{1}\right\|_{\infty}
\end{array}
$$

where:

$$
\begin{aligned}
C_{3} & :=C_{3}\left(p, \alpha, R_{0}\right):=N\left(K_{1} \frac{p-1}{p-\alpha-1}\right)^{p-1} R_{0}^{-1}, \\
C_{4} & :=C_{4}(a, b ; p, q ; \alpha, \beta, \gamma, \delta):=K_{1}^{\gamma} K_{2}^{\delta+1}, \\
C_{5} & :=C_{5}\left(R_{0} ; a ; p ; \alpha\right)=\frac{p-1}{p-\alpha-1} K_{1} R_{0}, \\
C_{5}^{\prime} & :=C_{5}\left(R_{0} ; b ; q ; \beta\right), \\
K_{1} & \left.:=K\left(\frac{a}{2}, p, \alpha\right) \text { and } K_{2}:=K\left(\frac{b}{2}, q, \beta\right) \text { (function } K \text { defined by }(2.1)\right) .
\end{aligned}
$$

We choose $X_{1}$ and $X_{2} \gg 1$ such that: $1+C_{5} X_{1}^{-1} \leq 2$ and $1+C_{5}^{\prime} X_{2}^{-1} \leq 2$, so (3.5) is satisfied if:

$$
\begin{aligned}
& C_{3} X_{1}^{\frac{(\alpha+1)(p-1)}{p-\alpha-1}} \geq 3\left\|f_{1}\right\|_{\infty}, \\
& C_{3} X_{1}^{\frac{(\alpha+1)(p-1)}{p-\alpha-1}} \geq 3 \times 2^{\delta+\gamma+1} c C_{4} X_{1}^{\frac{\gamma p}{p-\alpha-1}} X_{2}^{\frac{q(\delta+1)}{q-\beta-1}}, \\
& C_{3} X_{1}^{\frac{(\alpha+1)(p-1)}{p-\alpha-1}} \geq 3 \times 2^{\eta}\|\varphi\|_{\infty} K_{1}^{\eta} X_{2}^{\frac{q \eta}{q-\beta-1}} .
\end{aligned}
$$


Let $Z=X_{1}^{\frac{p(\gamma+1)}{p-\alpha-1}}=X_{2}^{\frac{q(\delta+1)}{q-\beta-1}}$ be large enough such that $Z \geq$ $\left(\frac{3}{C_{3}}\left\|f_{1}\right\|_{\infty}\right)^{\frac{(\gamma+1) p^{*}}{\alpha+1}}, c$ such that $c \leq \frac{C_{3}}{3 \times 2^{\gamma+\delta+1} C_{4}} Z^{\frac{\alpha+1}{(\gamma+1) p^{*}}-\frac{\gamma}{\gamma+1}-1}$ and $\|\varphi\|_{\infty} \leq \frac{C_{3}}{3 \times 2^{\eta} K_{2}^{n}} Z^{\frac{\alpha+1}{(\gamma+1) p^{*}}-\frac{\eta}{\delta+1}}$.

Similarly for the other equation of $(\mathcal{S})$, we take:

$$
Z \geq\left(\frac{3}{C_{3}^{\prime}}\left\|g_{1}\right\|_{\infty}\right)^{\frac{(\delta+1) q^{*}}{(\beta+1)}}, d \text { such that } d \leq \frac{C_{3}^{\prime}}{3 \times 2^{\gamma+\delta+1} C_{4}^{\prime}} Z^{\frac{\beta+1}{(\delta+1) q^{*}}-\frac{\delta}{\delta+1}-1}
$$

and $\|\psi\|_{\infty} \leq \frac{C_{3}^{\prime}}{3 \times 2^{\xi} K_{1}^{\xi}} Z^{\frac{\beta+1}{(\delta+1) q^{*}}-\frac{\xi}{\gamma+1}}$.

For (3.3) and (3.4), we take:

$$
\left\{\begin{array}{l}
c \leq C_{1} Z^{\frac{\alpha-2 \gamma-1}{\gamma+1}} \\
d \leq C_{1}^{\prime} Z^{\frac{\beta-2 \delta-1}{\delta+1}}
\end{array}\right.
$$

So, consider $Z$ large enough such that:

$$
Z \geq \operatorname{Max}\left(\left(\frac{2}{C_{3}}\left\|f_{1}\right\|_{\infty}\right)^{\frac{(\gamma+1)}{(\alpha+1) p^{*}}},\left(\frac{2}{C_{3}^{\prime}}\left\|g_{1}\right\|_{\infty}\right)^{\frac{(\delta+1)}{(\beta+1) q^{*}}}\right)
$$

and choose $c, d,\|\varphi\|_{\infty}$ and $\|\psi\|_{\infty}$ small enough such that:

$$
\begin{aligned}
0 & \leq c \leq \operatorname{Min}\left(\frac{C_{3}}{3 \times 2^{\delta+\gamma+1} C_{4}} Z^{\frac{\alpha+1}{(\gamma+1) p^{*}}-\frac{\gamma}{\gamma+1}-1}, C_{1} Z^{\frac{\alpha-2 \gamma-1}{\gamma+1}}\right), \\
0 & \leq d \leq \operatorname{Min}\left(\frac{C_{3}^{\prime}}{3 \times 2^{\delta+\gamma+1} C_{4}^{\prime}} Z^{\frac{\beta+1}{(\delta+1) q^{*}}-\frac{\delta}{\delta+1}-1}, C_{1}^{\prime} Z^{\frac{\beta-2 \delta-1}{\delta+1}}\right), \\
\|\varphi\|_{\infty} & \leq \frac{C_{3}}{3 \times 2^{\eta} K_{2}^{\eta}} Z^{\frac{\alpha+1}{(\gamma+1) p^{*}}-\frac{\eta}{\delta+1}}, \\
\|\psi\|_{\infty} & \leq \frac{C_{3}^{\prime}}{3 \times 2^{\xi} K_{2}^{\xi}} Z^{\frac{\beta+1}{(\delta+1) q^{*}}-\frac{\xi}{\gamma+1}} .
\end{aligned}
$$

Therefore, the existence of $(\hat{u}, \hat{v})$ in $\Omega$ is proved.

Theorem 1. Suppose that the hypothesis of Proposition 1 are satisfied, that $c, d,\|\varphi\|_{\infty}$ and $\|\psi\|_{\infty}$ are nonnegative real numbers sufficiently small. Then, there exists at least one nonnegative solution with compact support $(u, v)$ of problem $(\mathcal{S})$. 
Proof: We proceed in three steps.

i) Construction of an invariant set:

In view of applying Schauder's Fixed Point Theorem, let us introduce $E=L^{p}(\tilde{\Omega}) \times L^{q}(\tilde{\Omega}), K=[0, \hat{u}] \times[0, \hat{v}]$ and consider $m_{1}$ and $m_{2}$ such that $m_{1}>a$ and $m_{2}>b$.

Next we define the nonlinear operator $T:(u, v) \in K \rightarrow(w, z) \in E$ by:

$$
\begin{cases}-\Delta_{p} w+m_{1}|w|^{\alpha-1} w=\left(m_{1}-a\right) u^{\alpha}+f(x, u, v) & \text { in } \tilde{\Omega} \\ -\Delta_{p} z+m_{2}|z|^{\beta-1} z=\left(m_{2}-b\right) v^{\beta}+g(x, u, v) & \text { in } \tilde{\Omega} \\ w=z=0 & \text { on } \partial \tilde{\Omega}\end{cases}
$$

Existence and uniqueness for solutions of (3.10) are well-known by $[\mathbf{8}]$ and [4], so that $T$ is well defined. Moreover $(w, z)$ is in $W_{0}^{1, p}(\Omega) \cap L^{\infty}(\Omega) \times$ $W_{0}^{1, q}(\Omega) \cap L^{\infty}(\Omega)$ and is nonnegative by $[\mathbf{3}]$. Now we extend $w$ and $z$ by 0 out of $\tilde{\Omega}$.

ii) $T(K) \subset K$. Let $(u, v) \in K$, we have:

$$
\begin{aligned}
& -\left(\Delta_{p} w-\Delta_{p} \hat{\hat{u}}\right)+m_{1}\left(|w|^{\alpha-1} w-|\hat{u}|^{\alpha-1} \hat{u}\right) \leq \\
& \quad \leq\left(m_{1}-a\right)\left(|u|^{\alpha-1} u-|\hat{u}|^{\alpha-1} \hat{u}\right)+f(x, u, v)-f(x, \hat{u}, v),
\end{aligned}
$$

multiplying (3.11) by $\left.(w-\hat{u})_{+}:=\max (w-\hat{u}, 0)\right)$ and integrating over $\tilde{\Omega}$, we obtain:

$$
\begin{gathered}
\int_{\tilde{\Omega}}\left(\left|\nabla_{w}\right|^{p-2} \nabla w-|\nabla \hat{u}|^{p-2} \nabla \hat{u}\right) \nabla(w-\hat{u})_{+} d x+ \\
+m_{1} \int_{\tilde{\Omega}}\left(|w|^{\alpha-1} w-|\hat{u}|^{\alpha-1} \hat{u}\right)(w-\hat{u})_{+} d x \leq \\
\leq\left(m_{1}-a\right) \int_{\tilde{\Omega}}\left(u^{\alpha}-(\hat{u})^{\alpha}\right)(w-\hat{u})_{+} d x+ \\
+\int_{\tilde{\Omega}}(f(x, u, v)-f(x, \hat{u}, v))(w-\hat{u})_{+} d x \leq 0 .
\end{gathered}
$$

From $\left(\mathcal{H}_{1}\right)$ and Lemma 2 , we have $(w-\hat{u})_{+}=0$, hence $0 \leq w \leq \hat{u}$.

The same is true for $z, 0 \leq z \leq \hat{v}, T(K) \subset K$.

iii) $T$ is completely continuous:

First we prove that $T$ is compact, let $\left(u_{j}, v_{j}\right)$ be a bounded sequence in $K$. By $\left(\mathcal{H}_{2}\right) f\left(x, u_{j}, v_{j}\right)$ (resp. $g\left(x, u_{j}, v_{j}\right)$ ) is bounded in $L^{p^{*}}(\tilde{\Omega})$ (resp. $\left.L^{q^{*}}(\tilde{\Omega})\right)$. 
Multiplying the first equation in (3.10) by $w$, we obtain for $w_{j}$ :

$$
\begin{gathered}
\int_{\tilde{\Omega}}\left|\nabla w_{j}\right|^{p} d x+ \\
m_{1} \int_{\tilde{\Omega}}\left|w_{j}\right|^{\alpha+1} d x=\left(m_{1}-a\right) \int_{\tilde{\Omega}}\left|u_{j}\right|^{\alpha-1} u_{j} w_{j} d x+ \\
+\int_{\tilde{\Omega}} f\left(x, u_{j}, v_{j}\right) w_{j} d x \leq C\left(m_{1} \int_{\tilde{\Omega}}\left|w_{j}\right|^{p} d x\right)^{\frac{1}{p}}
\end{gathered}
$$

Hence $\left(w_{j}\right)$ is bounded in $W^{1, p}(\tilde{\Omega})$ and it possesses a strongly convergent subsequence in $L^{p}(\tilde{\Omega})$. The same is true for $z_{j}$ in $L^{q}(\tilde{\Omega})$.

Now we prove the continuity of $T$ :

Suppose that $\left(u_{j}, v_{j}\right) \rightarrow(u, v)$ in $K$. By the Dominated Convergence Theorem, we have:

$f\left(x, u_{j}, v_{j}\right) \rightarrow f(x, u, v)$ in $L^{p^{*}}(\tilde{\Omega})$ and $\left|u_{j}\right|^{\alpha-1} u_{j} \rightarrow|u|^{\alpha-1} u$ in $L^{p^{*}}(\tilde{\Omega})$.

\section{Consider}

(3.15)

$$
\begin{cases}-\left(\Delta_{p} w_{j}-\Delta_{p} w\right)+m_{1}\left(\left|w_{j}\right|^{\alpha-1} w_{j}-|w|^{\alpha-1} w\right)= & \\ =\left(m_{1}-a\right)\left(\left|u_{j}\right|^{\alpha-1} u_{j}-|u|^{\alpha-1} u\right)+f\left(x, u_{j}, v_{j}\right)-f(x, u, v) & \text { in } \tilde{\Omega} \\ w_{j}=w=0 & \text { on } \partial \tilde{\Omega} .\end{cases}
$$

Multiplying (3.15) by $\left(w_{j}-w\right)$ and integrating over $\tilde{\Omega}$ we obtain:

$$
\begin{gathered}
\int_{\tilde{\Omega}}\left(\left|\nabla w_{j}\right|^{p-2} \nabla w_{j}-|\nabla w|^{p-2} \nabla w\right) \nabla\left(w_{j}-w\right) d x+ \\
+m_{1} \int_{\tilde{\Omega}}\left(\left|w_{j}\right|^{\alpha-1} w_{j}-|w|^{\alpha-1} w\right)\left(w_{j}-w\right) d x= \\
=\left(m_{1}-a\right) \int_{\tilde{\Omega}}\left(\left|u_{j}\right|^{\alpha-1} u_{j}-|u|^{\alpha-1} u\right)\left(w_{j}-w\right) d x+ \\
+\int_{\tilde{\Omega}}\left(\left(f\left(x, u_{j}, v_{j}\right)-f(x, u, v)\right)\left(w_{j}-w\right) d x .\right.
\end{gathered}
$$

It follows from (3.14) that the right-hand side of (3.16) tend to zero as $j$ tends to $+\infty$.

From Lemma 2 and Holder's Inequality applied to the left-hand side of (3.16), we obtain:

$$
\int_{\tilde{\Omega}}\left|\nabla\left(w_{j}-w\right)\right|^{p} d x+m_{1} \int_{\tilde{\Omega}}\left|\left(w_{j}-w\right)\right|^{p} d x \rightarrow 0 \text { as } j \rightarrow+\infty .
$$


A similar argument can be used for $z$. Since $K$ is a convex, bounded and closed subset of $E$, we can apply Schauder's Fixed Point Theorem and obtain the existence of a fixed point for $T$, which gives the existence of at least one solution $(u, v)$ of $(\mathcal{S})$ such that $0 \leq u \leq \hat{u}$ and $0 \leq v \leq \hat{v}$.

\section{Parabolic systems}

In this section, we consider $p>2, q>2$ and $R_{0}>0$ such that:

$\operatorname{supp} \varphi \cup \operatorname{supp} \psi \cup \operatorname{supp} f_{1} \cup \operatorname{supp} g_{1} \cup \operatorname{supp} u_{0} \cup \operatorname{supp} v_{0} \subset B\left(R_{0}\right)$; where $f_{1}, g_{1}, \varphi, \psi$ defined in $\left(\mathcal{H}_{2}\right)$.

We add the following hypothesis:

$\left(\mathcal{H}_{3}\right) \forall M>0, \forall N>0, \exists K_{M, N}^{i}>0 i=1,2$ such that:

$$
\begin{aligned}
f\left(x, u_{1}, v_{1}\right)-f\left(x, u_{2}, v_{2}\right) & \leq K_{M, N}^{1}\left(\left(u_{1}-u_{2}\right)+\left(v_{1}-v_{2}\right)\right) ; \\
g\left(x, u_{1}, v_{1}\right)-g\left(x, u_{2}, v_{2}\right) & \leq K_{M, N}^{2}\left(\left(u_{1}-u_{2}\right)+\left(v_{1}-v_{2}\right)\right) \\
\text { for } 0 \leq u_{2} \leq u_{1} \leq M \text { and } 0 & \leq v_{2} \leq v_{1} \leq N .
\end{aligned}
$$

Proposition 2. Assume that the hypothesis $\left(\mathcal{H}_{0}\right)$ and $\left(\mathcal{H}_{1}\right)$ are satisfied and the numbers $\alpha, \beta, \gamma$ and $\delta$ are such that: $1 \leq \alpha<p-1$, $1 \leq \beta<q-1, \gamma \geq \alpha, \delta \geq \beta$. Then, for sufficiently small numbers $c, d,\|\varphi\|_{\infty}$ and $\|\psi\|_{\infty},(0,0)-(\hat{u}, \hat{v})$ is a sub-super solution of $(\mathcal{P})$.

Proof: From the definition of $\hat{u}$, for (II-b) it is sufficient to have:

$$
\left\|u_{0}\right\|_{\infty} \leq K_{1} X_{1}^{\frac{p}{p-\alpha-1}} \text {. }
$$

Similarly for $\hat{v}$,

$$
\left\|v_{0}\right\|_{\infty} \leq K_{2} X_{2}^{\frac{q}{q-\beta-1}},
$$

where $K_{1}:=K(a, p, \alpha), K_{2}:=K(b, q, \beta)$ (defined by $\left.(2.1)\right)$ and $X_{i}:=$ $R_{i}-R_{0}$ for $i=1,2$.

From the elliptic case (Proposition 1), (4.1) and (4.2), we choose the real numbers $Z, c, d,\|\varphi\|_{\infty}$ and $\|\psi\|_{\infty}$ such that:

$$
\begin{gathered}
Z \geq \operatorname{Max}\left(\left(\frac{2}{C_{3}}\left\|f_{1}\right\|_{\infty}\right)^{\frac{\gamma+1}{\alpha+1} \times p^{*}},\left(\frac{2}{C_{3}^{\prime}}\left\|g_{1}\right\|_{\infty}\right)^{\frac{\delta+1}{\beta+1} \times q^{*}},\right. \\
\left.\left(\frac{\left\|u_{0}\right\|_{\infty}}{K_{1}}\right)^{\gamma+1},\left(\frac{\left\|v_{0}\right\|_{\infty}}{K_{2}}\right)^{\delta+1}\right), \\
0 \leq c \leq \operatorname{Min}\left(\frac{C_{3}}{3 \times 2^{\delta+\gamma+1} C_{4}} Z^{\frac{\alpha+1}{(\gamma+1) p^{*}}-\frac{\gamma}{\gamma+1}-1}, C_{1} Z^{\frac{\alpha-2 \gamma-1}{\gamma+1}}\right), \\
0 \leq d \leq \operatorname{Min}\left(\frac{C_{3}^{\prime}}{3 \times 2^{\delta+\gamma+1} C_{4}^{\prime}} Z^{\frac{\beta+1}{(\delta+1) q^{*}}-\frac{\delta}{\delta+1}-1}, C_{1}^{\prime} Z^{\frac{\beta-2 \delta-1}{\delta+1}}\right),
\end{gathered}
$$


Then, the existence of $(\hat{u}, \hat{v})$ as super solution of $(\mathcal{P})$ in $Q_{T}$ is proved.

Theorem 2. Assume that $\left(\mathcal{H}_{3}\right)$ and the hypothesis of Proposition 2 are satisfied. Then the problem $(\mathcal{P})$ admits a nonnegative unique solution in $\mathcal{C}\left(\mathbb{R}^{+}, L^{2}(\Omega)\right) \cap L^{\infty}\left(0, T ; W_{0}^{1, p}(\Omega)\right) \times \mathcal{C}\left(\mathbb{R}^{+}, L^{2}(\Omega)\right) \cap L^{\infty}\left(0, T ; W_{0}^{1, q}(\Omega)\right)$ such that: $0 \leq u(x, t) \leq \hat{u}(x)$ and $0 \leq v(x, t) \leq \hat{v}(x)$ in $\Omega \times \mathbb{R}^{+}$.

Proof: Using an iterative method, we proceed in five steps.

Construction of sequence $\left(\underline{u}_{n}\right)$ (resp. $\left.\left(\underline{v}_{n}\right)\right)$.

i) Determination of $\underline{u}_{0}$ (resp. $\underline{v}_{0}$ ).

From [6, Theorem 4] $\underline{u}_{0}$ (resp. $\underline{v}_{0}$ ) exists as solution of:

$$
\begin{cases}\frac{\partial \underline{u}_{0}}{\partial t}-\Delta_{p} \underline{u}_{0}+a\left|\underline{u}_{0}\right|^{\alpha-1} \underline{u}_{0}=f(x, 0,0) & \text { in } \tilde{Q}_{T}:=\tilde{\Omega} \times[0, T] \\ \underline{u}_{0}(x, t)=0 & \text { on } \tilde{\Sigma}_{T}:=\partial \tilde{\Omega} \times[0, T] \\ \underline{u}_{0}(., 0)=u_{0}(.) & \text { in } \tilde{\Omega},\end{cases}
$$

resp.

$$
\begin{cases}\frac{\partial \underline{v}_{0}}{\partial t}-\Delta_{q} \underline{v}_{0}+b\left|\underline{v}_{0}\right|^{\beta-1} \underline{v}_{0}=g(x, 0,0) & \text { in } \tilde{Q}_{T} \\ \underline{v}_{0}(x, t)=0 & \text { on } \tilde{\Sigma} \tilde{v}_{T} \\ \underline{v}_{0}(., 0)=v_{0}(.) & \text { in } \tilde{\Omega},\end{cases}
$$

such that: $0 \leq \underline{u}_{0} \leq \hat{u}\left(\right.$ resp. $\left.0 \leq \underline{v}_{0} \leq \hat{v}\right)$.

ii) Suppose $\left(\underline{u}_{n}\right)$ (resp. $\left.\left(\underline{v}_{n}\right)\right)$ is defined as nonnegative function on $\bar{\Omega} \times[0, T](T>0)$ with initial value $u_{0}$ (resp. $\left.v_{0}\right)$ in $\Omega$, zero on $\tilde{\Sigma}_{T}$ and $0 \leq \underline{u}_{n} \leq \hat{u}$ (resp. $0 \leq \underline{v}_{n} \leq \hat{v}$ ) also we define $\underline{u}_{n+1}$ (resp. $\underline{v}_{n+1}$ ) as solution of the problem:

$$
\begin{cases}\frac{\partial \underline{u}_{n+1}}{\partial t}-\Delta_{p} \underline{u}_{n+1}+a\left|u_{n+1}\right|^{\alpha-1} u_{n+1}=f\left(x, \underline{u}_{n}, \underline{v}_{n}\right) & \text { in } \tilde{Q}_{T} \\ \underline{u}_{n+1}(x, t)=0 & \text { on } \tilde{\Sigma}_{T} \\ \underline{u}_{n+1}(., 0)=u_{0}(.) & \text { in } \tilde{\Omega},\end{cases}
$$

resp.

$$
\begin{cases}\frac{\partial \underline{v}_{n+1}}{\partial t}-\Delta_{q} \underline{v}_{n+1}+b\left|\underline{v}_{n+1}\right|^{\beta-1} \underline{v}_{n+1}=g\left(x, \underline{u}_{n}, \underline{v}_{n}\right) & \text { in } \tilde{Q}_{T} \\ \underline{v}_{n+1}(x, t)=0 & \text { on } \tilde{\Sigma}_{T} \\ \underline{v}_{n+1}(., 0)=v_{0}(.) & \text { in } \tilde{\Omega} .\end{cases}
$$

$\underline{u}_{n+1}$ (resp. $\underline{v}_{n+1}$ ) exists by [6, Theorem 4] such that: $0 \leq \underline{u}_{n+1} \leq \hat{u}$ $\left(\right.$ rsp. $0 \leq \underline{v}_{n+1} \leq \hat{v}$ ). 
iii) Estimations of $\underline{u}_{n+1}, \frac{\partial \underline{u}_{n+1}}{\partial t}$ (resp. $\underline{v}_{n+1}, \frac{\partial \underline{v}_{n+1}}{\partial t}$ ).

Consider:

$$
\begin{cases}\frac{\partial \underline{u}_{n+1}(x, t)}{\partial t}-\Delta_{p} \underline{u}_{n+1}+a\left|\underline{u}_{n+1}\right|^{\alpha-1} \underline{u}_{n+1}=f\left(x, \underline{u}_{n}, \underline{v}_{n}\right) & \text { in } \tilde{Q}_{T} \\ \underline{u}_{n+1}(x, t)=0 & \text { on } \tilde{\Sigma} \\ \underline{u}_{n+1}(., 0)=u_{0}(.) & \text { in } \tilde{\Omega} .\end{cases}
$$

Multiplying by $\frac{\partial \underline{u}_{n+1}}{\partial t}$ and integrating over $\tilde{Q}_{T}$, we get:

$$
\begin{aligned}
& \int_{\tilde{Q}_{T}}\left(\frac{\partial \underline{u}_{n+1}(x, t)}{\partial t}\right)^{2}-\int_{\tilde{Q}_{T}} \Delta_{p} \underline{u}_{n+1} \frac{\partial \underline{u}_{n+1}(x, t}{\partial t} d x d t+ \\
+ & a \int_{\tilde{Q}_{T}}\left|\underline{u}_{n+1}\right|^{\alpha-1} \underline{u}_{n+1} \frac{\partial \underline{u}_{n+1}(x, t)}{\partial t} d x d t= \\
= & \int_{\tilde{Q}_{T}} f\left(x, \underline{u}_{n}, \underline{v}_{n}\right) \frac{\partial \underline{u}_{n+1}(x, t)}{\partial t} d x d t,
\end{aligned}
$$

then

$$
\begin{gathered}
\int_{\tilde{Q}_{T}}\left(\frac{\partial \underline{u}_{n+1}(x, t)}{\partial t}\right)^{2} d x d t+\frac{1}{p} \int_{\tilde{\Omega}}\left|\nabla \underline{u}_{n+1}(x, T)\right|^{p} d x+ \\
+\frac{a}{\alpha+1} \int_{\tilde{\Omega}}\left|\underline{u}_{n+1}(x, T)\right|^{\alpha+1} d x \leq c \int_{\tilde{Q}_{T}} \underline{u}_{n}^{\gamma} \underline{v}_{n}^{\delta+1} d x d t+ \\
+\int_{\tilde{Q}_{T}} \varphi(x) \underline{v}_{n}^{\eta} \frac{\partial \underline{u}_{n+1}(x, t)}{\partial t} d x d t+\int_{\tilde{Q}_{T}} f_{1}(x) \frac{\partial \underline{u}_{n+1}(x, t)}{\partial t} d x d t+ \\
+\frac{1}{p} \int_{\tilde{\Omega}}\left|\nabla u_{0}(x)\right|^{p} d x+\frac{a}{\alpha+1} \int_{\tilde{\Omega}}\left|u_{0}(x)\right|^{\alpha+1} d x .
\end{gathered}
$$

Using Young's Inequality, we have:

$$
\begin{aligned}
\left\|\frac{\partial \underline{u}_{n+1}(x, t)}{\partial t}\right\|_{L^{2}\left(\tilde{Q}_{T}\right)} & \leq C(T) \\
\left\|\underline{u}_{n+1}\right\|_{L^{\infty}\left(0, T ; W_{0}^{1, p}(\tilde{\Omega})\right)} & \leq C(T) \\
\left\|\underline{u}_{n+1}\right\|_{L^{\infty}\left(\tilde{Q}_{T}\right)} & \leq C(T),
\end{aligned}
$$

where $C(T)$ is a positive constant. 
From (4.3), (4.4) and (4.5), there exists a subsequence which converges to $u$ in the sense of weak $*$ topology in $L^{\infty}\left(0, T ; W_{0}^{1, p}(\tilde{\Omega})\right)$, which converges also to $u$ weakly in $L^{p}\left(0, T ; W_{0}^{1, p}(\tilde{\Omega})\right)$ and $\frac{\partial \underline{u}_{n+1}}{\partial t}$ converges weakly to $\frac{\partial u}{\partial t}$ in $L^{2}\left(\tilde{Q}_{T}\right)$.

By the argument of monotonicity [8], $\Delta_{p} \underline{u}_{n}$ converges weakly to $\Delta_{p} u$ in $L^{p^{*}}\left(0, T ; W_{0}^{-1, p^{*}}(\tilde{\Omega})\right)$. Since $\underline{u}_{n} \rightarrow u$ a.e. and $\underline{v}_{n} \rightarrow v$ a.e., then we have by Convergence Dominated Theorem: $\underline{u}_{n+1}^{\gamma} \underline{v}_{n}^{\delta+1}$ converges to $u^{\gamma} v^{\delta+1}$.

Hence $(u, v)$ is solution of $(\mathcal{P})$ in $\tilde{Q}_{T}$ such that:

$$
0 \leq u(x, t) \leq \hat{u}(x) \text { and } 0 \leq v(x, t) \leq \hat{v}(x) .
$$

We extend $u$ by 0 and $v$ by 0 out of $\tilde{\Omega} \times[0, T](\forall T>0)$.

v) Uniqueness: Suppose that there exists $\left(u_{1}, u_{2}\right)$ and $\left(v_{1}, v_{2}\right)$ two solutions of problem $(\mathcal{P})$, we have:

$\frac{\partial\left(u_{1}-u_{2}\right)}{\partial t}-\left(\Delta_{p} u_{1}-\Delta_{p} u_{2}\right)+a\left(u_{1}^{\alpha}-u_{2}^{\alpha}\right)=f\left(x, u_{1}, v_{1}\right)-f\left(x, u_{2}, v_{2}\right)$,

multiplying by $\left(u_{1}-u_{2}\right)$, we obtain by using the monotonicity of operator $-\Delta_{p}$ and $\left(\mathcal{H}_{3}\right)$ :

$$
\begin{aligned}
\frac{1}{2} \frac{\partial}{\partial t} \int_{\tilde{\Omega}}\left(u_{1}-u_{2}\right)^{2}(x, t) d x & d t \leq K_{B_{1}, B_{2}}^{1} \int_{\tilde{\Omega}}\left(u_{1}-u_{2}\right)^{2}(x, t) d x d t+ \\
+ & K_{B_{1}, B_{2}}^{1} \int_{\tilde{\Omega}}\left(u_{1}-u_{2}\right)\left(v_{1}-v_{2}\right)(x, t) d x d t .
\end{aligned}
$$

Similarly:

$$
\begin{aligned}
\frac{1}{2} \frac{\partial}{\partial t} \int_{\tilde{\Omega}}\left(v_{1}-v_{2}\right)^{2}(x, t) d x & d t \leq K_{B_{1}, B_{2}}^{2} \int_{\tilde{\Omega}}\left(v_{1}-v_{2}\right)^{2}(x, t) d x d t+ \\
+ & K_{B_{1}, B_{2}}^{2} \int_{\tilde{\Omega}}\left(u_{1}-u_{2}\right)\left(v_{1}-v_{2}\right)(x, t) d x d t
\end{aligned}
$$

where the constants $K_{B_{1}, B_{2}}^{1}$ and $K_{B_{1}, B_{2}}^{2}$ are positive constant; where $B_{1}$ and $B_{2}$ are defined in (2.2).

By Holder's Inequality, we get:

$$
\begin{aligned}
& \frac{1}{2} \frac{\partial}{\partial t}\left[\left\|\left(u_{1}-u_{2}\right)(x, t)\right\|_{L^{2}(\tilde{\Omega})}^{2}+\left\|\left(v_{1}-v_{2}\right)(x, t)\right\|_{L^{2}(\tilde{\Omega})}^{2}\right] \leq \\
& \leq C\left[\left\|\left(u_{1}-u_{2}\right)(x, t)\right\|_{L^{2}(\tilde{\Omega})}^{2}+\left\|\left(v_{1}-v_{2}\right)(x, t)\right\|_{L^{2}(\tilde{\Omega})}^{2}\right],
\end{aligned}
$$

where $C$ is a positive constant. 
Then from Gronwall's Lemma, we obtain: $u_{1}=u_{2}$ and $v_{1}=v_{2}$.

If $p>\frac{2 N}{N+2}$ or $p=N, W^{1, p}(\Omega) \hookrightarrow L^{2}(\Omega)$ and as $u \in L^{\infty}\left(0, T ; W_{0}^{1, p}(\Omega)\right)$ and $\frac{\partial u}{\partial t} \in L^{2}\left(Q_{T}\right)$ then from Lemma 3 we have $u \in \mathcal{C}\left(\mathbb{R}^{+}, L^{2}(\Omega)\right)$ and similarly for $v \in \mathcal{C}\left(\mathbb{R}^{+}, L^{2}(\Omega)\right)$.

\section{References}

1. ARIs, H., "The Mathematical Theory of Diffusion and Reaction in Permeable Catalysts," Volumes 1 and 2, Clarendon Press, Oxford, 1975.

2. DíAz, J. I., "Nonlinear Partial Differential Equations and Free Boundaries," Volume 1, Research Notes in Mathematics, Pitman, 1985.

3. DíAz, J. I. AND HERnÁNDEZ J, On the existence of a free boundary for a class of reaction-diffusion systems, SIAM J. Math. Anal. 15(4) (1989), 670-685.

4. Díaz, J. I. And Herrero M. A., Propriétés de support compact pour certaines equations elliptiques et paraboliques non linéaires, $C$. R. Acad. Sci. Paris 286 (1978), 815-817.

5. Díaz, J. I. ANd Herrero M. A., Estimates on the support of the solutions of some nonlinear elliptic and parabolic problems, Proc. Roy. Soc. of Edinburgh 89A (1981), 249-258.

6. El Hachimi, A. And De Thelin, F., Supersolutions and stabilization of the solutions of the equation $\left(\frac{\partial u}{\partial t}\right)-\operatorname{div}\left(|\nabla u|^{p-2} \nabla u\right)=f(x, u)$, Nonlinear Anal. 12 (1988), 1385-1398.

7. HeRnÁNDEZ, J., "Qualitative methods for nonlinear diffusion equations," Lecture Notes in Math. 1224, Springer-Verlag, 1985, pp. 47-118.

8. Lions, J. L., "Quelques Méthodes de Résolution des Problèmes aux Limites Non Linéaires," Dunod, Paris, 1969.

9. NAKaO, M., On a system of nonlinear diffusion equations, Funkcialaj Ekvacioj 27 (1984), 75-84.

10. Simon, J., Régularité de la Solution d'une Equation Non Linéaire dans $\mathbb{R}^{N}$, in "Journées d'Analyse Non Linéaire," Ph. Benilan and J. Robert, eds., Lecture Notes in Math. 665, Springer-Verlag, Berlin, 1978, pp. 205-227. 
11. De Thelin, F., Résultats d'existence et de non-existence pour la solution positive et bornée d'une E.D.P. elliptique non linéaire, $A n$ nales Fac. Sc. Toulouse 8 (1986-87), 375-389.

\author{
Laboratoire M.I.P. \\ Université Paul Sabatier \\ 31062 Toulouse Cedex \\ FRANCE
}

Rebut el 5 de Novembre de 1993 